ALCOHOL-RELATED INHIBITORY CONTROL

Note: This paper is a pre-print version of a manuscript published in Experimental \& Clinical Psychopharmacology, and is not the final copy-edited version.

\title{
Alcohol Belongs Here: Assessing Alcohol-related Inhibitory Control with a Contextual Go/No-Go Task
}

Charlotte R. Pennington ${ }^{1 *}(\mathrm{PhD})$, Rebecca L. $\operatorname{Monk}^{2}(\mathrm{PhD})$, Adam W. Qureshi $^{2}(\mathrm{PhD})$ ' \& Derek Heim² $(\mathrm{PhD})$

${ }^{1}$ Department of Health and Social Sciences, University of the West of England, Coldharbour Lane, Bristol, BS16 1QY

${ }^{2}$ Edge Hill University, Department of Psychology, St Helens Road, Ormskirk, Lancashire, L39 4QP

*Corresponding Author: Charlotte R. Pennington, E-mail: charlotte.pennington@uwe.ac.uk ${ }^{1}$ Department of Health and Social Sciences, University of the West of England, Coldharbour Lane, Bristol, BS16 1QY

Open Data Statement: Data can be viewed on the Lead Author's OSF profile: https://osf.io/x9m4q/

Tables: 4

Figures: 3 
ALCOHOL-RELATED INHIBITORY CONTROL

\begin{abstract}
There is a growing awareness of the need to explore the social and environmental milieus that drive alcohol consumption and related cognitions. The current study examined the extent to which alcohol-congruent and incongruent drinking contexts modulate alcohol-related inhibitory control using a novel Go/No-Go task. One-hundred and eight participants (Mage = 20 years; $S D=4.87$ ) were instructed to inhibit their responses to visual alcoholic (Alcohol/NoGo condition, $n=50$ ) or non-alcoholic stimuli (Alcohol/Go condition, $n=58$ ) depicted in an alcohol-congruent (pub), incongruent (library) or context free (control) condition. Participants in the Alcohol/Go condition exhibited higher false alarm rate (FAR) towards non-alcoholic stimuli and faster reaction times (RT) to alcoholic stimuli depicted in the alcohol-congruent and incongruent context compared to the Alcohol/No-Go condition. In contrast, FAR towards alcoholic stimuli (Alcohol/No-Go condition) were not significantly affected by drinking context but RT was faster when non-alcoholic stimuli were presented in an alcohol-incongruent (i.e., library) compared to alcohol-congruent context (i.e., pub). The discussion turns to potential explanations for these findings, suggesting that social drinkers might exhibit approach tendencies towards alcoholic images that translate into errors towards non-alcoholic stimuli, and that image complexity influences response inhibition.
\end{abstract}

Key words: Alcohol consumption; Go/No-Go task; inhibitory control; social context; environment 
ALCOHOL-RELATED INHIBITORY CONTROL

\section{Public Significance Statement}

The current study examines the influence of environmental drinking context on social drinker's ability to withhold responses to alcoholic cues. Findings suggest that individuals who drink alcohol may show an automatic approach bias towards alcoholic stimuli depicted in images of real world contexts when they are not under the influence of alcohol. Furthermore, the complexity of images employed within alcohol research (e.g., context vs. no context) might influence alcohol-related response inhibition.

\section{Author Note}

The data within this manuscript have not been published elsewhere, in whole or in part, and have not been presented at any academic conferences.

\section{Disclosures and Acknowledgements}

No funding is associated with this research.

All listed authors contributed significantly to this manuscript and approve its submission for publication. Specifically, all authors contributed to the study design; CRP and AWQ collected and analysed the data, with checks performed by RLM and DH; CRP wrote the first draft of the manuscript with revisions made by RLM, AWQ and DH.

The authors declare no conflicts of interest.

Every person who aided assistance and contributed to this research are listed as authors on this manuscript. 


\section{Introduction}

When people think about consuming alcohol, they may not only envisage the characteristics of the drink, but also contextual factors such as their social drinking environment and associated sights, sounds and smells. Indeed, there is a growing awareness that contextual factors play a significant role in driving alcohol cue reactivity and consumption behaviours (Thrul, Labhart, \& Kuntsche, 2017; see Heim \& Monk, 2017 for commentary). The influence that different social milieus exert on alcohol behaviours is illustrated by research indicating that alcohol consumption is greater in social groups relative to drinking alone (e.g., Kuendig \& Kuntsche, 2012; Kuntsche, Kuntsche, Thrul, \& Gmel, 2017; Monk et al., 2015; 2017a; Thrul \& Kuntsche, 2015) and when one's group size is larger (e.g., Thrul et al., 2017; Thrul \& Kuntsche, 2015). Moreover, consumption is less well remembered retrospectively when drinking takes place in a pub compared to at home (Monk, Heim, Qureshi \& Price, 2015). One explanation for this pattern of findings is that the presence of other people and the desire to socialise may enhance the positive effects of alcohol, which in turn enriches the value of associated social stimuli (de Wit \& Sayette, 2018).

Research also demonstrates how social and environmental context can influence the cognitions underpinning alcohol consumption behaviours. Social factors appear to exert variable effects on self-reported alcohol-related expectancies (Labrie, Grant, \& Hummer, 2011; Monk \& Heim, 2013a; Wall, McKee, \& Hinson, 2000; Wiers et al., 2003), normative beliefs (McAlaney, Bewick, \& Bauerle, 2010; Pedersen, Labrie, \& Lac, 2008), drinking motives (Anderson, Garcia, \& Dash, 2017; Halim, Hasking, \& Allen, 2012), and craving (Jones, Rose, Cole, \& Field, 2013b; Mason, Light, Escher, \& Drobes, 2008; Trela et al., 2018). For example, Wall et al. (2000) found that people reported greater stimulation, perceived dominance and pleasurable disinhibition when tested in a bar compared to a laboratory setting, suggesting that alcohol outcome expectancies may be modulated by cue exposure. Similarly, research has 
ALCOHOL-RELATED INHIBITORY CONTROL

found that alcohol-related outcome expectancies become more positive in naturalistic social settings (Labrie et al., 2011; Monk \& Heim, 2013b) and drink refusal self-efficacy reduces in the presence of other social drinkers (Monk et al., 2013a). A recent study indicates further that individuals with low sensitivity to alcohol exhibit elevated momentary alcohol cravings in environments that closely resemble their typical drinking situation (Trela et al., 2018). Implicit cognitions related to alcohol-related beliefs also appear to vary significantly when alcoholic stimuli are depicted in social drinking scenes (Monk et al., 2016a). Here, participants more readily endorsed positive alcohol-related expectancies when alcohol was depicted in a congruent (i.e., pub) compared to incongruent drinking environment (i.e., lecture theatre) and this was strengthened when participants were situated in a pub (versus a lecture theatre - Monk et al., 2016a).

Likewise, a wealth of research has investigated context effects by examining how people respond to alcohol-related cues on experimental tasks (termed 'cue reactivity'; see Field \& Cox, 2008 for a review). This work indicates consistently that both social and problem drinkers exhibit impairments in inhibitory control when required to inhibit responses towards visual alcoholic relative to non-alcoholic stimuli (Christiansen, Cole, \& Field, 2012; Kreusch et al., 2013; 2017; Weafer \& Fillmore, 2015), with this effect found to be modulated by alcoholrelated sounds (Qureshi et al., 2017) and smells (Monk et al., 2016b). Nevertheless, it is also important to note that other research casts doubt on the extent of these effects (see Jones et al., 2018a). Rather than being a stable trait, inhibitory control may therefore be a limited resource that can be contextually depleted (Muraven \& Baumeister, 2000; though see Leotti \& Wager, 2009 for a trait approach), particularly when it comes to addictive behaviours (De Wit, 2009; Jones et al., 2013a; 2013b). In support of this, a recent study demonstrates how transient dayto-day variations in inhibitory control present as a risk factor for alcohol consumption (Jones et al., 2018b). 
ALCOHOL-RELATED INHIBITORY CONTROL

Alcohol-related cue reactivity highlights how contexts that are associated with consumption can elicit conditioned responses of individuals (Carter \& Tiffany, 1999; see Field $\&$ Cox, 2008 for a review). A conditioned appetitive response consists of a 'wanting' response which is implicated in approach behaviours (i.e., incentive salience; Robinson \& Berridge, 1993; see also Peeters et al., 2012; Wiers et al., 2010), and conditioned contexts have been shown to modify the effect of alcohol-related cues on such responses (Nees et al., 2012; see also Trela et al., 2018). Supporting this assertion, alcohol-dependent patients rate social drinking contexts and full alcohol beverage containers as more pleasant and arousing relative to neutral drinking situations and empty glasses/bottles (Nees et al., 2012). In addition, alcoholseeking behaviour in animals emerges when conditioning is conducted in an environment where alcohol has been consumed previously relative to a non-alcohol-related environment (Sciascia et al., 2015). Associative learning principles therefore seem to account for the effects of presentation context on alcohol-related cognitions and associated consumption.

Past research has shaped our understanding of the importance of alcohol-related cues and their associative contexts in the expression of conditioned responses. However, there are certain methodological limitations inherent in past work, which we argue may have prevented a systematic exploration of context effects. Specifically, Nees et al. (2012) compared participants' subjective ratings of craving, valence and arousal when viewing pictures of social and neutral environments and compared responses between scenes depicting alcoholic beverages and no beverage. Consequently, there was no control condition with a non-alcoholic beverage. Similarly, Monk et al. (2016a) examined implicit alcohol-related expectancies by asking participants to match positive and negative statements to alcoholic beverages depicted in the foreground of a pub or university lecture theatre. These stimuli provided a default association between alcohol and context (e.g., a beer bottle in the foreground of a bar or lecture theatre) and did not provide a neutral condition (e.g., a soft drink in the same context; Kuntsche 
ALCOHOL-RELATED INHIBITORY CONTROL

\& Kuntsche 2017). Moreover, previous research has examined contextual influences on alcohol-related inhibitory control typically by measuring participants' responses to single images of alcoholic and non-alcoholic beverages in experimental paradigms (e.g., Petit et al., 2012; Pennington, Qureshi, Monk, \& Heim, 2016; Qureshi et al., 2017), and some have utilised unmatched, non-appetitive control stimuli (e.g., household objects; see Duka \& Townshend, 2008; Kreusch et al., 2013; Kvamme et al., 2018; Jones \& Field, 2015). However, this limits the investigation of broader contextual influences (i.e., one's drinking situation, different available beverages, which may diminish inhibitory control further through the process of associative conditioning.

Building on past research, the current study investigated the influence of drinking context on alcohol-related cue reactivity, with an explicit focus on inhibitory control. Specifically, it assessed whether the contextual presentation of (non) alcoholic stimuli has the capacity to modulate alcohol-related inhibitory control. In a novel approach, we adapted a Go/No-Go (GNG) task to depict alcoholic and non-alcoholic pictorial stimuli in varying background contexts (i.e., alcohol-congruent - a pub; alcohol-incongruent - a library; controlno context). Consistent with prior research (e.g., Christiansen et al., 2012; Kreusch et al., 2013; 2017; Weafer \& Fillmore, 2015), it was predicted that participants would exhibit higher false alarm rate (i.e., FAR - indicative of impaired inhibitory control) when instructed to inhibit responses to alcoholic (Alcohol/No-Go condition) relative to non-alcoholic stimuli (Alcohol/Go condition). Further, we hypothesised that inhibitory control would be diminished to a greater extent when alcoholic stimuli were depicted in an alcohol-congruent (pub) compared to an alcohol-incongruent drinking context (library). Similarly, it was predicted that participants would respond quicker to alcoholic drinks presented in an alcohol-congruent context (beer - pub) and non-alcoholic drinks presented in an alcohol-incongruent context (water - library). 
ALCOHOL-RELATED INHIBITORY CONTROL

\section{Method}

\section{Participants and Design}

The experiment consisted of a 2 (Target stimuli: Alcohol/No-Go, Alcohol/Go) x 3 (Background context: Alcohol-congruent $=$ Pub, incongruent $=$ library, control $=$ no context $)$ mixed-design, with target stimuli as the between-participants factor meaning that some participants were instructed to inhibit responses to alcohol-related stimuli (Alcohol/No-Go condition) and others to non-alcohol stimuli (Alcohol/Go). An a-priori power analysis (G*Power; Faul et al., 2009) indicated that a total sample size of 86 participants across the two conditions was required to detect a moderate effect size $(f=0.25)$ with $80 \%$ statistical power. Participants were excluded if they reported not drinking alcohol $(n=10$; AUDIT $=0)$, or because of unmatched responses between their pre-questionnaire responses and GNG data $(n=13$, i.e., forgotten or mistyped memorable numbers). After these exclusions, the final sample consisted of 108 participants recruited through study sign-ups $(M a g e=20.50, S D=4.87$, range $=18-51 ; 86$ female; $79.6 \%$

White British) from a UK university. Participants were assigned randomly to either the Alcohol/No-Go $(n=50)$ or Alcohol/Go condition $(n=58)$ and received course credits or equivalent monetary remuneration for their time. All data, including exclusions, can be viewed at: https://osf.io/x9m4q/

\section{Measures and Materials}

Go/No-Go Task (GNG)

The GNG task (Nosek \& Banaji, 2001) comprised four blocks, each consisting of 144 test trials ( $n=576$ test trials in total). Eighteen additional practice trials were included but removed from the final analyses. Within each block there were 40 'Go' trials and eight 'No-Go' trials for each background context (alcohol-congruent, incongruent, none): Go-trials occurred frequently $(83.33 \%)$ to influence a pre-potent tendency for participants to respond. Go-stimuli were alcohol-related or non-alcohol-related beverages, which corresponded to the random allocation 
ALCOHOL-RELATED INHIBITORY CONTROL

of participants to two experimental conditions (Alcohol/No-Go or Alcohol/Go). Each trial started with a fixation point presented for randomly varied durations (stimulus onset asynchrony; 100-500ms) followed by either a 'Go' or 'No-Go' trial. The inter-trial interval was $700 \mathrm{~ms}$, and stimuli were presented for $1000 \mathrm{~ms}$, or until the participant responded. Table 1 provides an overview of the experimental design.

\section{[TABLE 1 HERE]}

Pictorial stimuli of branded alcoholic and non-alcoholic beverages were selected from the Amsterdam Beverage Picture Set (Pronk et al., 2015) - an inventory of matched alcoholic and non-alcoholic products, which has been validated across cultures (see Boffo, Pronk, Wiers, \& Mannarani, 2015). Four pictures of different branded beer bottles and four pictures of different branded water bottles were utilised for the target stimuli based on equivalent brand familiarity. These target images were then superimposed onto three background images: An alcohol-congruent context (a pub), an alcohol-incongruent context (a library) and a no context control condition. This produced a total set of 24 image types, with 12 containing the four different alcoholic targets and 12 containing the four non-alcoholic targets shown in each of the three contexts. See Figure 1 for example trial types. All component images (both stimulus and context), as well as the final test images, were matched for size and luminosity and were displayed randomly within each block. Table 2 provides the internal reliability for each trial type; Cronbach's $a$ values of $>0.60$ are considered acceptable (see Williams \& Kaufman, 2012) and all values are consistent with prior research using the original GNG task (e.g., Qureshi et al., 2017). As a measure of inhibitory control, we extracted the proportion of trials in which the participant made an incorrect response to a No-Go stimulus, providing a measure of false alarm rate (FAR), as well as accuracy and reaction times (RT) for 'Go' trials.

[FIGURE 1 HERE] 
ALCOHOL-RELATED INHIBITORY CONTROL

[TABLE 2 HERE]

Alcohol Use Disorders Identification Test (AUDIT)

The AUDIT (Saunders et al., 1993) was employed to measure participants' alcohol consumption, drinking behaviour and alcohol-related problems. Responses to this 10-item questionnaire are recorded on a 5-point Likert scale anchored between 0 (Never) and 5 (Almost always) and then summed. A score of 8 or more indicates harmful drinking patterns. This measure resulted in acceptable internal consistency, Cronbach's $\alpha=.71$, with a sample mean of $7.69(S D=4.08)$ indicating that our sample consisted generally of moderate social drinkers.

\section{Adult Temperament Questionnaire (ATQ)}

The effortful control (EC) sub-scale of the ATQ (Evans \& Rothbart, 2007; Rothbart, Ahadi, \& Evans, 2000) was employed to assess participants' perceived ability to regulate their behaviour; a trait that has been found to moderate alcohol consumption behaviours (see Gerich, 2014; Robinson, Jones, Christiansen, \& Field, 2014; Qureshi et al., 2017). ). This 35-item questionnaire incorporates three sub-scales assessing inhibitory control (i.e., capacity to inhibit approach tendencies), activation control (i.e., ability to perform an action when there is a strong tendency to avoid it) and attentional control (i.e., focusing and shifting attention). Participants respond to questions such as "It's often hard for me to alternate between two different tasks" on a Likert scale anchored between 1 (Extremely untrue of you) and 7 (Extremely true of you) with a mean score computed. This measure also resulted in acceptable internal consistency, $\alpha$ $=.76$, with a sample mean of $4.18(S D=.51)$.

\section{Procedure}

Ethical approval for this study was obtained from the relevant institutional governing bodies (REF: HAS.17.09.018 Pennington, “Contextual effects in an alcohol GNG task”). Participants 
ALCOHOL-RELATED INHIBITORY CONTROL

completed the AUDIT and ATQ questionnaires before arriving at the laboratory to complete the GNG Task to avoid alcohol-related priming (see Melaugh-McAteer, Curran, \& Hanna, 2015), and memorable dates were recorded to match participants' questionnaire responses with their task performance. Participants were tested in a group laboratory and assigned randomly to either the Alcohol/No-Go or Alcohol/Go condition, with those tested in each session assigned to the same experimental condition to prevent demand characteristics. Throughout the task, participants wore headphones and dividers obstructed the view of other participant's computer screens. During the GNG task, participants in the Alcohol-No/Go condition were instructed that, irrespective of the background context, they should respond to non-alcoholic target stimuli (bottled water; Go-trials) as quickly as possible by hitting the space bar on a standard keyboard. On the remaining No-Go trials, they were instructed to inhibit responses to alcoholic target stimuli (bottled beers). Those in the Alcohol-Go condition received the same instructions but, conversely, were asked to respond to alcoholic beverages during Go-trials and inhibit their responses to non-alcoholic stimuli during No-Go trials. Breaks were provided between each block to reduce fatigue. After completion of the experiment, participants received a full verbal and written debrief, which also included information relating to local and national alcohol-related support services.

\section{Analytic Strategy}

A 2 (Target stimuli: Alcohol/No-Go, Alcohol/Go) x 3 (Background Context: Congruent = pub, incongruent $=$ library, control $=$ none $)$ mixed-design Analysis of Variance $($ ANOVA) was conducted on False Alarm Rate (FAR), Go-trial reaction times (RT) and Go-trial accuracy. Target stimuli was entered as a between-participants factor and background context as a withinparticipants factor. Greenhouse-Geisser corrections are reported when the assumption of sphericity is violated. Bonferroni-corrections are applied to elucidate simple main effects and interactions, with effect sizes denoted by Cohen's $d$ (see Cohen, 1992). Mixed-design 
ALCOHOL-RELATED INHIBITORY CONTROL

ANCOVAs were then conducted to examine whether self-reported alcohol consumption (AUDIT) or trait effortful control (ATQ) explained any of the variance in our initial findings. Specifically, AUDIT scores were included as a covariate in accordance with research demonstrating the association between heavy drinking and deficits in response inhibition (see Field et al., 2010 for review). Further, research suggests that trait effortful control may be inversely related to alcohol consumption behaviours (Gerich, 2014; Robinson et al., 2014; Qureshi et al., 2017), and thus, those who score higher on this trait may be better able to inhibit prepotent responding towards alcohol-related stimuli. Covariates were mean-centred to aid interpretability.

\section{Results}

\section{Preliminary Analyses}

Independent $t$-tests indicated that participants in the Alcohol/No-Go and Alcohol/Go conditions did not significantly differ in terms of AUDIT or ATQ scores. This provided assurance that any differences between conditions could be attributed to the experimental task, rather than individual differences in alcohol consumption or trait effortful control between participants. Additional checks were then conducted to ensure that AUDIT,ATQ scores and GNG performance did not significantly differ between males and females in our sample (all $p$ $>$.23). Moreover, age did not significantly correlate with GNG performance for any of the trial types. See Table 3 for descriptive statistics.

[TABLE 3 HERE]

\section{FAR}


ALCOHOL-RELATED INHIBITORY CONTROL

There was a significant main effect of background context on $\operatorname{FAR}, F(1.81,191.77)=23.97, p$ $<.001, \eta_{p}^{2}=.18$. Simple main effects indicated that FAR was significantly higher for the alcohol-congruent context $\left(M_{\text {pub }}=.11, S D=.11\right)$ compared to no context $(M=.07, S D=.08)$, $p<.001, d=.42$. FAR was also significantly higher for the alcohol-incongruent context $(M$ library $=.13, S D=.13$ ) compared to no context, $p<.001, d=.56$. There was no significant difference, however, between FAR in the alcohol-congruent and incongruent context, $p=.07$, $d=.17$. A main effect of target stimuli indicated that FAR was significantly higher for nonalcoholic stimuli (Alcohol/Go, $M=.15, S D=.08$ ) compared to alcoholic stimuli (Alcohol/NoGo; $M=.06, S D=.09), \underline{F}(1,106)=27.71, p<.001, \eta_{p}^{2}=.21$. This was qualified by a significant two-way interaction between target stimuli and background context, $F(1.81$, 191.77) $=26.13, p<.001, \eta_{p}^{2}=.20$. Contrary to predictions, participants in the Alcohol/Go condition exhibited higher FAR towards non-alcoholic images depicted in both the alcoholcongruent and alcohol-incongruent drinking context $\left(M_{\text {pub }}=.17, S D=.12 ; M_{\text {library }}=.18, S D\right.$ $=.15)$ compared to the Alcohol/No-Go condition $\left(M_{\text {pub }}=.05, S D=.05 ; M_{\text {library }}=.07, S D=\right.$ $.07)$, both $p<.001, d=1.31$ and .94 respectively. There was no significant difference, however, in FAR towards alcoholic (Alcohol/No-Go; $\left.M_{\text {control }}=.06, S D=.08\right)$ and non-alcoholic images $($ Alcohol/Go, $M$ control $=.08, S D=.08)$ depicted in no context, $p=.16$. For those in the Alcohol/Go condition, FAR was significantly higher for both the alcohol-congruent $\left(M_{\mathrm{pub}}=\right.$ $.17, S D=.12)$ and alcoholic-incongruent contexts $\left(M_{\text {library }}=.18, S D=.15\right)$ compared to no context $(M=.08, S D=.08)$, both $p<.001, d=.88$ and .83 , respectively. There were no significant differences in the Alcohol/No-Go condition as a function of context, $p>.05$. See Figure 2 for interaction. Including AUDIT or ATQ did not remove any of the main effects of interactions.

[FIGURE 2 HERE] 
ALCOHOL-RELATED INHIBITORY CONTROL

\section{Go-trial RT}

A significant main effect of background context indicated that participants were faster to respond to stimuli depicted within no context $(M=417.52, S D=52.51)$ followed by the alcohol-incongruent $\left(M_{\text {library }}=423.13, S D=54.20\right)$ and alcohol-congruent context $\left(M_{\text {pub }}=\right.$ 427.89, $S D=54.15), F(2,212)=41.11, p<.001, \eta_{p}^{2}=.28$ (all differences significant, $p<$ $.001)$. There was a significant main effect of target stimuli $F(1,106)=9.61, p=.002, \eta_{p}^{2}=$ .08 ; with quicker responses to alcoholic stimuli (Alcohol/Go condition; $M=408.73, S D=$ 50.95) compared to non-alcoholic stimuli (Alcohol/No-Go condition; $M=439.22, S D=50.98$ ), $p=.002, d=.60$. There was also a significant two-way interaction between target stimuli and background context, $F(2,212)=34.46, p<.001, \eta_{p}^{2}=.25$. Participants in the Alcohol-Go condition responded to alcoholic stimuli depicted in an alcohol-congruent $\left(M_{\mathrm{pub}}=409.80, S D\right.$ $=50.26)$ and alcohol-incongruent context $\left(M_{\text {library }}=407.78, S D=51.44\right)$ significantly faster than those in the Alcohol/No-Go condition $\left(M_{\text {pub }}=448.87, S D=51.26 ; M_{\text {library }}=440.93, S D\right.$ $=52.30$ ), both $p<.01, d=.77$ and .80 , respectively. Responses to target stimuli did not differ significantly, however, when there was no background context, $p=.06, d=.04$. Participants in the Alcohol/No-Go condition responded quicker to non-alcoholic stimuli (i.e., water) presented in no context $(M=427.85, S D=54.16)$, followed by the alcohol-incongruent context $\left(M_{\text {library }}\right.$ $=440.93, S D=52.30)$ and alcohol-congruent context $\left(M_{\text {pub }}=448.87, S D=51.26\right)$, all $p<.001$, $d$ ranging from .15-.40. There were no significant differences in RT between background contexts for participants in the Alcohol/Go condition $(p>.33)$. See Figure 3 for interaction. These findings remained when AUDIT and ATQ scores were included as covariates. 
[FIGURE 3 HERE]

\section{Go-trial Accuracy}

There was no significant main effect of context $(p=.056)$ or target stimuli $(p=.12)$, and no two-way interaction between context and target stimuli $(p=.53)$. These findings remained when AUDIT and ATQ were included as covariates. The lack of any significant effects observed here is likely due to Go-trial accuracy being high across both conditions and all blocks (.99). Table 4 presents descriptive statistics for all dependent variables.

\section{[TABLE 4 HERE]}

\section{Discussion}

The current study investigated whether the contextual presentation of alcoholic stimuli has the capacity to modulate alcohol-related inhibitory control. Using a novel GNG task, participants were instructed to inhibit their responses to either alcoholic (Alcohol/No-Go) or non-alcoholic target stimuli (Alcohol/Go) depicted in an alcohol-congruent (pub) or incongruent (library) environmental context, or no context (control). Contrary to predictions, participants in the Alcohol/Go condition were less able to inhibit their responses to non-alcoholic stimuli depicted in both the alcohol-congruent (pub) and alcohol-incongruent drinking context (library) compared to no context. Furthermore, they exhibited higher FAR towards non-alcoholic stimuli presented in the alcohol-congruent and incongruent context compared to participants in the Alcohol/No-Go condition, whose responses towards alcoholic stimuli were not significantly modulated by drinking context. Such findings are interesting because they appear to contradict previous research showing that individuals who consume alcohol display impaired inhibitory control towards alcohol-related relative to neutral stimuli (e.g., 
ALCOHOL-RELATED INHIBITORY CONTROL

Christiansen et al., 2012; Kreusch et al., 2013; 2017; Weafer \& Fillmore, 2015). However, prior studies examining inhibitory control have not manipulated environmental context, which has been recognised increasingly as a driver of alcohol consumption and related cognitions (Thrul et al., 2017; see also Heim \& Monk, 2017).

These results may tentatively suggest that participants in the Alcohol/Go condition exhibited an excitatory approach response towards alcoholic stimuli depicted in different environmental contexts, which translated into poorer inhibition towards non-alcoholic stimuli on No-Go trials. This appears to be supported by the finding that these participants responded more quickly to Go-trials depicting alcoholic stimuli in alcohol-congruent and alcoholincongruent contexts relative to those in the Alcohol/No-Go condition. This speed-accuracy trade off could reflect attentional bias towards alcohol (see Field \& Cox, 2008; Kreusch et al., 2017), which has been found to be heightened by expectations of drug availability (Field et al., 2011; Jones et al., 2012; 2018b; Pronk et al., 2015). As such, the frequency of alcoholic stimuli presented in the Alcohol/Go condition (to evoke a prepotent response) may have increased the motivational salience of alcohol, leading to approach biases and increased errors towards nonalcoholic stimuli.

Support for this assertion comes from models of incentive salience (Robinson \& Berridge, 1993; see also Peeters et al., 2012; Wiers et al., 2010), which theorise that a conditioned appetitive response consists of a 'wanting' response driving approach behaviours. Underpinned by this, a wealth of research suggests that individuals are more likely to respond to alcohol with an approach movement (i.e., pulling a joystick) on the Alcohol Approach-Avoid Task (AAT; Wiers, Rinck, Dictus, \& Van den Wildenberg, 2009), and are quicker to associate alcohol with approach compared to avoidance terms on the Alcohol Approach-Avoid Implicit Association Test (IAT; see Wiers et al., 2009; 2010). With this in mind, our findings may suggest that repeatedly responding to alcoholic cues within a congruent context may evoke 
ALCOHOL-RELATED INHIBITORY CONTROL

approach tendencies, possibly by activating associations between alcohol and one's typical drinking environment (see Trela et al., 2018). Such interpretation is nevertheless speculative and future research would benefit from examining whether the current findings converge with behavioural responses on tasks capable of measuring approach tendencies (e.g., IAT, AAT), and other measures indicative of wanting behaviours (e.g., elevated subjective craving).

Relatedly, it is plausible that instructing participants in the Alcohol/No-Go condition to respond repeatedly to non-alcoholic stimuli strengthened their ability to inhibit responses towards alcoholic stimuli. Indeed, a recent study found that pairing empty glasses with a gocue and beer-related pictures with a no-go cue effectively reduces positive automatic associations for alcohol and subsequent alcohol intake (Houben, Havermans, Nederkoon, \& Jansen, 2012). Similar training protocols have also been shown to be effective for alcoholdependent patients (Wiers, Rinck, Kordts, Houben, \& Strack, 2010; Wiers, Erberl, Rinck, Becker, \& Lindenmeyer, 2011). Although our paradigm did not pair responding with a stop signal, participants in the Alcohol/No-Go condition responded to non-alcoholic stimuli during approximately $80 \%$ of consecutive trials (total trial $n=576$ ). Continuously responding to nonalcoholic stimuli depicted in different drinking contexts may have therefore increased respondents' ability to inhibit alcohol-related responding.

When considering the above explanations, however, it should be emphasised that the current results were obtained only when comparing responses between the alcohol-congruent (pub) and incongruent (library) drinking contexts. That is, there was no significant difference in FAR between alcohol-related and neutral stimuli in the no context condition. This may add weight to the notion that researchers should consider contextual factors in the study of alcoholrelated processes more routinely (see Thrul et al., 2018; Heim \& Monk, 2018). Nevertheless, the authors also acknowledge that there was a difference in image complexity between the alcohol-congruent and alcohol-incongruent conditions relative to the control condition. 
ALCOHOL-RELATED INHIBITORY CONTROL

Although contextual images might be more representative of real world drinking environments, these are also more complex because they feature visually rich scenes that might influence cognitive processing. Consistent with this, research indicates that social drinkers show attentional bias towards simple but not complex alcohol-related stimuli (e.g., single alcoholic bottles relative to a crowded bar scene), with this effect possibly transferring across different tasks that measure alcohol-related cognitions (Miller et al., 2010). Examination of RT in the current study does, however, indicate that participants in the Alcohol/No-Go condition appeared able to distinguish between the drinking contexts, exhibiting quicker responses towards non-alcoholic stimuli depicted in a congruent (library) compared to a non-congruent drinking context (pub). More generally, participants responded quicker to images displayed in the no context condition, followed by the alcohol-incongruent (library) and alcohol-congruent conditions (pub).

Moreover, the authors note that the results from the control condition are unlikely to generalise readily to the real world; it is hard to envisage a situation whereby alcohol-related cues are present with no context. Aiding theoretical explication, it is not uncommon in this area of research to draw comparisons between responses to singular presented stimuli that are visually divergent from alcohol-related stimuli. For example, research typically examines alcohol-related inhibitory control by presenting single alcohol-related or non-alcoholic neutral items within different experimental paradigms (e.g., Petit et al., 2012; Pennington et al., 2016; Qureshi et al., 2017). Past research also pinpoints inhibitory failures by comparing alcoholrelated stimuli with neutral, non-appetitive stimuli (e.g., stationary, household items, and alphabetical letters; see Duka \& Townshend, 2008; Kreusch et al., 2013; Jones \& Field, 2015; Pennington et al., 2016). This study and other emerging research (see Monk et al., 2017b) may therefore highlight that the types of stimuli used may have an important bearing on findings. 


\section{Limitations and Future Directions}

This study is the first to explore whether environmental drinking context modulates inhibitory control by incorporating alcohol-congruent and incongruent backgrounds into a novel GNG Task. Here we build on past research by utilising matched alcoholic and non-alcoholic appetitive stimuli and varying the congruency of the drinking environment. Nevertheless, it is important to acknowledge a number of potential limitations pertaining to our study design, sample and findings.

We arguably utilised a more complex design than previous studies employing to the GNG task but despite this, FAR was very low towards alcohol-related stimuli (.05-.07 across contexts), which contrasts with prior studies where inhibitory control failures are typically higher (e.g., Kreusch et al., 2013; Qureshi et al., 2017). One potential explanation for this is that the current sample consisted mainly of moderate social drinkers (AUDIT; $M=7.69, S D=$ 4.08) who may be better able to regulate their responses towards alcohol compared to heavier drinkers. Furthermore, $92 \%$ of our sample were aged between 18-24 years, and recent UK national statistics indicate that young people in this age group are now less likely to drink regularly compared to any other age group (Office for National Statistics, 2018). Considering that inhibitory control mechanisms have been found to differ as a function of drinking status (Kreusch et al., 2013; Roberts et al., 2014; Wiers et al., 2002), one future avenue for research would be to employ the current paradigm to compare responses between light and heavy drinkers or clinical and non-clinical populations.

There were no significant differences in age, AUDIT scores or trait effortful control between participants in the Alcohol/Go and Alcohol/No-Go conditions, thus providing assurance that differences in GNG performance were not be attributable to the recorded sample 
ALCOHOL-RELATED INHIBITORY CONTROL

characteristics. Nevertheless, there may be additional unmeasured variables that account for the unexpected findings between the Alcohol/Go and Alcohol/No-Go condition. For example, we assessed self-reported trait effortful control prior to the experimental testing phase but did not employ a baseline cognitive measure of inhibitory control performance. Research points to a weak relationship between explicit reports of effortful control and cognitive tasks, with each independently predicting everyday impulsive behaviours (see Sharma et al., 2014). It could therefore be prudent for future research to include pre-test measures of inhibitory control, particularly when utilising between-participant designs. In addition, we did not assess whether participants had any diagnosis of clinical psychopathology, such as ADHD, which may impact inhibitory control (see Wright, Lipszyc, Dupuis, Thayapararajah, \& Schachar, 2014). We believe that the random allocation of participants to the two conditions, as well as the relatively large sample sizes in each condition, will have controlled for this to a large extent. Nevertheless, future research would benefit from investigating how individual differences contribute to alcohol consumption and related cognitions (i.e., inhibitory control, attentional bias), particularly considering that this is a largely under-researched area.

We utilised four different branded alcoholic and four different branded non-alcoholic target stimuli from a validated battery of images (ABI; Pronk et al., 2015) that were matched for familiarity. Research has shown, however, that the visual characteristics of branding may influence cognitive bias (Kersbergen \& Field, 2017), and it is therefore possible that different results would emerge when using non-branded products. Furthermore, we selected only one type of beverage (i.e., various pictures of beer and water) and it is conceivable that different findings may emerge if multiple alcoholic and non-alcoholic stimuli were to be included in the design, or if participants' personal drinking preferences were accounted for. Indeed, research suggests that people who consume beer and spirits tend to consume more alcohol and may be at greater risk of alcohol-related problems than people who drink other alcoholic beverages 
ALCOHOL-RELATED INHIBITORY CONTROL

(Gronbaek, Jensen, Johansen, Sørensen, \& Becker, 2004; , Siegel, Naimi, Cremeens, \& Nelson, 2011). Further, alcoholic beverage choice is underpinned by differences in drinking motives (i.e., enhancement, social, conformity, coping; Kuntsche, 2001; Kuntsche, Knibbe, Gmel, \& Engels, 2006). Future studies may therefore benefit from measuring whether people's drinking preference influences their response inhibition towards particular drinks or generalises across different alcoholic stimuli.

To examine fully the effect of environmental context, our task could be used in an ecological momentary assessment of alcohol-related inhibitory control (e.g., a matched 2 [pictorial context; pub/library] x 2 [in-vivo context; pub/library] design). Recent research reveals that inhibitory control is malleable; individuals associate alcohol more strongly with positive outcome expectancies when they are situated in a pub environment (Monk et al., 2016a), and daily fluctuations in inhibitory control predict subsequent alcohol consumption (Jones et al., 2018b). From this perspective, it is possible that inhibitory control may be diminished further in environments where alcohol is readily available (i.e., pubs and bars). One next step would therefore be to examine whether the current paradigm is capable of measuring fluctuations in inhibitory control dependent on experimental manipulations of context (e.g., alcohol depicted in a pub) and one's current drinking environment (i.e., being situated in a pub). Such investigations would advance our understanding of how in-vivo drinking context influences alcohol-related cognitions.

\section{Conclusions}

There is a growing awareness that social and environmental milieus exert an important influence on alcohol consumption behaviours (see Thrul et al., 2017; Heim \& Monk, 2017). Underpinned by this, the current study examined the impact of alcohol-congruent and incongruent drinking contexts on inhibitory control. The findings indicate that participants 
ALCOHOL-RELATED INHIBITORY CONTROL

assigned to the Alcohol/Go condition displayed higher FAR towards non-alcoholic stimuli depicted in an alcohol congruent and incongruent context compared to no context. Moreover, these same participants had greater FAR towards non-alcoholic stimuli and responded quicker to alcoholic stimuli depicted in an alcohol congruent and incongruent context compared to those in the Alcohol/No-Go condition. Such finding may suggest that participants in the Alcohol/Go condition exhibit an automatic approach bias towards alcohol on Go-trials, which impairs response inhibition towards non-alcoholic stimuli on No-Go trials. This might reflect the incentive salience of alcohol to these social drinkers. Conversely, it is plausible that responding repeatedly to non-alcoholic stimuli (Alcohol/No-Go condition) might train response inhibition towards alcoholic stimuli. Future research that employs our task alongside a measure of alcohol approach tendencies would represent a prudent step towards supporting or refuting this assertion.

At first glance, these findings appear to contrast with previous research; however, studies have not manipulated drinking context in the assessment of alcohol-related inhibitory control (e.g., Christiansen et al., 2012; Kreusch et al., 2013; 2017; Weafer \& Fillmore, 2015). Moreover, some compareresponses towards alcohol-related stimuli with unmatched nonappetitive stimuli (e.g., Duka \& Townshend, 2008; Kreusch et al., 2013; Kvamme et al., 2018; Jones \& Field, 2015; Pennington et al., 2016). It is therefore possible that previous research exaggerates the effect of alcohol-related stimuli on inhibitory control by studying visual stimuli in relative isolation from other contextual cues that might contribute to an individuals' ability to suppress prepotent responses. 
ALCOHOL-RELATED INHIBITORY CONTROL

\section{Figure Legends}

Figure 1. Example trial-types; contextually congruent (left), incongruent (middle) and no context (right).

Figure 2. Two-way interaction between target stimuli (between-participants factor) and background context (within-participants factor) on FAR. Error bars represent standard errors and $*$ denotes statistical significance, all at $p<.001$.

Figure 3. Two-way interaction between target stimuli and background context on Go-trial RT. Error bars represent standard errors and $*$ denotes statistical significance, all at $p<.001$. 
ALCOHOL-RELATED INHIBITORY CONTROL

\section{References}

Anderson, K. G., Garcia, T. A., \& Dash, G. F. (2017). Drinking motives and willingness to drink alcohol in peer drinking contexts. Emerging Adulthood, 5, 16-26. doi:10.1177/2167696816636503

Boffo, M., Pronk, T., Wiers, R. W., \& Mannarani, S. (2015). Combining cognitive bias modification training with motivational support in alcohol dependent outpatients: Study protocol for a randomised controlled trial. Trials, 16, 63. doi: 10.1186/s13063-015-05766

Carter, B. L., \& Tiffany, S. T. (1999). Meta-analysis of cue-reactivity in addiction research. Addiction, 94, 327-340. doi:10.1046/j.1360-0443.1999.9433273.x

Christiansen, P., Cole, J. C., \& Field, M. (2012). Ego depletion increases ad-lib alcohol consumption: Investigating cognitive mediators and moderators. Experimental and Clinical Psychopharmacology, 20, 118-128. doi:10.1037/a0026623

Cohen, J. (1992). A power primer. Psychological Bulletin, 112, 155-159. doi:10.1111/14678721.ep10768783

de Wit, H. (2009). Impulsivity as a determinant and consequence of drug use: A review of underlying processes. Addiction Biology, 14, 22-31. doi:10.1111/j.13691600.2008.00129.x

de Wit, H., \& Sayette, M. (2018). Considering the context: social factors in responses to drugs in humans. Psychopharmacology, 1-11. doi:10.1007/s00213-018-4854-3

Duka, T., \& Townshend, J. M. (2004). The priming effect of alcohol pre-load on attentional bias to alcohol-related stimuli. Psychopharmacology, 176, 353-361. doi:10.1007/s00213-004-1906-7 
ALCOHOL-RELATED INHIBITORY CONTROL

Evans, D. E., \& Rothbart, M. K. (2007). Development of a model for adult temperament. Journal of Research in Personality, 41, 868-888. doi:10.1016/j.jrp.2006.11.002

Faul, F., Erdfelder, E., Buchner, A., \& Lang, A. G. (2009). Statistical power analyses using G*Power 3.1: Tests for correlation and regression analyses. Behavioural Research Methods, 41, 1149-1160. doi:10.3758/BRM.41.4.1149

Field, M., \& Cox, W. M. (2008). Attentional bias in addictive behaviors: A review of its development, causes, and consequences. Drug and Alcohol Dependence, 97, 1-20. doi:10.1016/j.drugalcdep.2008.03.030

Field, M., Wiers, R. W., Christiansen, P., Fillmore, M. T., \& Verster, J. C. (2010). Acute alcohol effects on inhibitory control and implicit cognition: Implications for loss of control over drinking. Alcoholism Clinical \& Experimental Research, 34, 1346-1352. doi:10.1111/j.1530-0277.2010.01218.x

Gerich, J. (2014). The inhibiting function of self-control and social control on alcohol consumption. Journal of Drug Issues, 44, 120-131.

Gronbaek, M., Jensen, M. K., Johansen, D., Sørensen, T. I. A., \& Becker, U. (2004). Intake of beer, wine and spirits and risk of heavy drinking and alcoholic cirrhosis. Biological Rsearch, 37, 195-200. doi:10.4067/S0716-97602004000200004

Halim, A., Hasking, P., \& Allen, F. (2012). The role of social drinking motives in the relationship between social norms and alcohol consumption. Addictive Behaviors, 12, 1335-1341. doi:10.1016/j.addbeh.2012.07.004

Heim, D., \& Monk, R. L. (2017). Commentary on Thrul et al (2017): A welcome step towards a more context-aware addiction science. Addiction, 112, 440-441. doi:10.1111/add.13713 
ALCOHOL-RELATED INHIBITORY CONTROL

Houben, K., Havermans, R. C., Nederkoorn, C., \& Jansen, A. (2012). Beer à no-go: Learning to stop responding to alcohol cues reduces alcohol intake via reduced associations rather than increased response inhibition. Addiction, 107, 1280-1287. doi:10.1111/j.13600443.2012.03827.x

Jones, A., \& Field, M. (2015). Alcohol-related and negatively valenced cues increase motor and oculomotor disinhibition in social drinkers. Experimental \& Clinical Psychopharmacology, 23, 122-129. doi:10.1037/pha0000011

Jones, A., Hogarth, L., Christiansen, P., Rose, A. K., Martinovic, J., \& Field, M. (2012). Reward expectancy promotes generalized increases in attentional bias for rewarding stimuli. Quarterly Journal of Experimental Psychology, 65, 2333-2342. doi:10.1080/17470218.2012.686513

Jones, A., Christiansen, P., Nederkoorn, C., Houben, K., \& Field, M. (2013a). Fluctuating disinhibition: Implications for the understanding and treatment of alcohol and other substance use disorders. Frontiers in Psychiatry, 22, 140. doi:10.3389/fpsyt.2013.00140

Jones, A., Rose, A. K., Cole, J., \& Field, M. (2013b). Effects of alcohol cues on craving and ad libitum alcohol consumption in social drinkers: The role of disinhibition. Journal of Experimental Psychopathology, 4, 239-249. doi:10.37/pha0000011

Jones, A., Robinson, E., Duckworth, J., Kersbergen, I., Clarke, N., \& Field, M. (2018a). The effects of exposure to appetitive cues on inhibitory control: A meta-analytic investigation. Appetite, 128, 271-282. doi:10.1016/j.appet.2018.06.024

Jones, A., Tiplady, B., Houben, K., Nederkoorn, C., \& Field, M. (2018b). Do daily fluctuations in inhibitory control predict alcohol consumption? An ecological momentary assessment study. Psychopharmacology, 1-10. doi:10.1007/s00213-018-4860-5 
ALCOHOL-RELATED INHIBITORY CONTROL

Kersbergen, I., \& Field, M. (2017). Alcohol consumers' attention to warning labels and brand information on alcohol packaging: Findings from cross-sectional and experimental studies. BMC Public Health, 17, 123. doi:10.1186/s12889-017-4055-8

Kreusch, F., Billieux, J., \& Quertemont, E. (2017). Alcohol-cue exposure decreases response inhibition towards alcohol-related stimuli in detoxified alcohol-dependent patients. Psychiatry Research, 249, 232-239. doi:10.1016/j.psychres.2017.01.019

Kreusch, F., Villenne, A., \& Quertemont, E. (2013). Response inhibition toward alcoholrelated cues using an alcohol go/no-go task in problem and non-problem drinkers. Addictive Behaviors, 38, 2520-2528. doi:10.1016/j.addbeh.2013.04.007

Kuendig, H., \& Kuntsche, E. (2012). Solitary versus social drinking: An experimental study on effects of social exposures on in-situ alcohol consumption. Alcoholism: Clinical and Experimental Research, 36, 732-738. doi:10.1111/j.1530-0277.2011.01663.x

Kuntsche, E. (2001). Radikalisierung? Ein interpretations modell jugendlichen Alkoholkonsums von 1986 bis 1998 in der Schweiz (Radicalisation? An interpretation mofel of adolescent alcohol use from 1986 to 1998 in Switzerland). Sucht, 47, 393-403.

Kuntsche, E., \& Kuntsche, S. (2017). Development and initial validation of the Alcohol Expectancy Task. Alcoholism: Clinical and Experimental Research, 41, 1461-1470. doi: 10.1111/acer.13427

Kuntsche, E., Knibbe, R., Gmel, G., \& Engels, R. (2006). 'I drink spirits to get drunk and block out my problems...' Beverage preference, drinking motives and alcohol use in adolescence. Alcohol \& Alcoholism, 41, 566-573. doi:10.1093/alcalc/ag1046

Kuntsche, E., Kuntsche, S., Thrul, J., \& Gmel, G. (2017). Binge drinking: Health impact, prevalence, correlates and interventions. Psychology \& Health, 32, 976-1017. doi:10.1080/08870446.2017.1325889 
ALCOHOL-RELATED INHIBITORY CONTROL

Kvamme, T. L., Thomsen, K. R., Callesen, M. B., Doñamayor, N., Jensen, M., Pedersen, M. U., \& Voon, V. (2018). Distraction towards contextual alcohol cues and craving are associated with levels of alcohol use among youth. BMC psychiatry, 18(1), 354. doi: $\underline{10.1186 / \mathrm{s} 12888-018-1919-0}$

LaBrie, J. W., Grant, S., \& Hummer, J. F. (2011). “This would be better drunk": Alcohol expectancies become more positive while drinking in the college social environment. Addictive Behaviors, 36, 890-893. doi:10.1016/j.addbeh.2011.03.015

Leotti, L. A., \& Wager, T. D. (2009). Motivational influences on response inhibition. Journal of Experimental Psychology: Human Perception \& Performance, 36, 430-447. doi:10.1037/a0016802

Mason, B. J., Light, J. M., Escher, T., \& Drobes, D. J. (2008). Effect of positive and negative affective stimuli and beverage cues on measures of craving in treatment-seeking alcoholics. Psychopharmacology, 200, 141-150. doi:10.1007/s00213-008-1192-x

McAlaney, J., Bewick, B. M., \& Bauerle, J. (2010). Social norms guidebook: A guide to implementing the social norms approach in the UK. University of Bradford, University of Leeds, Department of Health: West Yorkshire, UK.

Melaugh McAteer, A., Curran, D., \& Hanna, D. (2015). Alcohol attention bias in adolescent social drinkers: An eye tracking study. Psychopharmacology, 232, 3183-3191. doi:10.1007/s00213-015-3969-z

Miller, M. A., \& Fillmore, M. T. (2010). The effect of image complexity on attentional bias towards alcohol-related images in adult drinkers. Addiction, 105, 883-890. doi:10.1111/j.1360-0443.2009.02860.x

Monk, R. L., \& Heim, D. (2013a). Panoramic projection; affording a wider view on contextual influences on alcohol-related cognitions. Experimental and Clinical Psychopharmacology, 21, 1-7. doi:10.1037/a0030772 
ALCOHOL-RELATED INHIBITORY CONTROL

Monk, R. L., \& Heim, D. (2013b). Environmental context effects on alcohol-related outcome expectancies, efficacy and norms: A field study. Psychology of Addictive Behaviors, 27, 814-818. doi:10.1037/a0033948

Monk, R. L., Heim, D., Qureshi, A., \& Price, A. (2015). "I have no clue what I drunk last night": Using smartphone technology to compare in-vivo and retrospective self-reports of alcohol consumption. PLoS One, 10(5), e0126209

Monk, R. L., Pennington, C. R., Campbell, C., Price, A., \& Heim, D. (2016a). Implicit alcohol-related expectancies and the effect of context. Journal of Studies on Alcohol and Drugs, 77, 819-827. doi:10.15288/jsad.2016.77.819

Monk, R. L., Sunley, J., Qureshi, A. W., \& Heim, D. (2016b). Smells like inhibition: The effects of olfactory and visual alcohol cues on inhibitory control. Psychopharmacology, 233, 1331-1337. doi:10.1007/s00213-016-4221-1

Monk, R. L., Qureshi, A. W., McNeill, A., Erskine-Shaw, M., \& Heim, D. (2017a). Perfect for a gin and tonic: How context drives consumption within a modified bogus taste test. Alcohol and Alcoholism, 9, 1-7. doi:10.1093/alcalc/agx084

Monk, R. L., Qureshi, A. Pennington, C. R., \& Hamlin, I. (2017b). Generalised inhibitory impairment to appetitive cues: From alcoholic to non-alcoholic stimuli. Drug and Alcohol Dependence, 180, 26-32. doi:10.1016/j.drugalcdep.2017.07.038

Muraven, M., \& Baumeister R. F. (2000). Self-regulation and depletion of limited resources: Does self-control resemble a muscle? Psychological Bulletin, 126, 247-259. doi:10.1037//0033-2909.126.2.247

Nees, F., Diener, C., Smolka, M. N., \& Flor, H. (2012). The role of context in the processing of alcohol-relevant cues. Addiction Biology, 17, 441-51. doi:10.1111/j.13691600.2011.00347.x 
ALCOHOL-RELATED INHIBITORY CONTROL

Nosek, B. A., \& Banaji, M. R. (2001). The Go/No-Go Association Task. Social Cognition, $19,625-664$.

Office for National Statistics. (2018). Adult drinking habits in Great Britain: 2017. Statistical Bulletin. Available from:

https://www.ons.gov.uk/peoplepopulationandcommunity/healthandsocialcare/drugusealco holandsmoking/bulletins/opinionsandlifestylesurveyadultdrinkinghabitsingreatbritain/2005 $\underline{\text { to2016 }}$

Pedersen, E. R., Labrie, J. W., \& Lac, A. (2008). Assessment of perceived and actual alcohol norms in varying contexts: Exploring social impact theory among college students. Addictive Behaviors, 33, 552-564. doi:10.1016/j.addbeh.2007.11.003

Pennington, C. R., Qureshi, A., Monk, R. L., \& Heim, D. (2016). The effects of stereotype threat and contextual cues on alcohol users' inhibitory control. Addictive Behaviors, 54, 12-17. doi:10.1016/j.addbeh.2015.11.014

Peeters, M., Wiers, R. W., Monshouwer, K., van de Schoot, R., Janssen, T., \& Vollebergh, W. A. (2012). Automatic processes in at-risk adolescents: the role of alcohol-approach tendencies and response inhibition in drinking behavior. Addiction, 107, 1939-1946. doi:10.1111/j.1360-0443.2012.03948.x

Petit, G., Kornreich, C., Noël, X., Verbanck, P., \& Campanella, S. (2012). Alcohol-related context modulates performance of social drinkers in a visual Go/No-Go task: A preliminary assessment of event-related potentials. PloS One, 7(5), e37466. doi:10/1371/journal.pone.0037466

Pronk, T., van Deursen, D. S., Beraha, E. M., Larsen, H., \& Wiers, R. W. (2015). Validation of the Amsterdam Beverage Picture Set: A controlled picture set for cognitive bias measurement and modification paradigms. Alcohol Clinical and Experimental Research, 39, 2047-2055. doi:10.1111/acer.12853. 
ALCOHOL-RELATED INHIBITORY CONTROL

Qureshi. A. W., Monk, R. L., Pennington, C. R., Li, X., \& Leatherbarrow, T. (2017). Context and alcohol consumption behaviours affect inhibitory control. Journal of Applied Social Psychology, 47, 625-633. doi:10.1111/jasp.12465

Roberts, W., Miller, M. A., Weafer, J., \& Fillmore, M. T. (2014). Heavy drinking and the role of inhibitory control of attention. Experimental and Clinical Psychopharmacology, 22, 133-140. doi:10.1037/a0035317

Robinson, E., Jones, A., Christiansen, P., \& Field, A. (2014). Drinking like everyone else: Trait self-control moderates the association between peer and personal heavy episodic drinking. Substance Use \& Misuse, 50, 1532-2491, doi:10.3109/10826084.2014.991407

Robinson, T. E., \& Berridge, K. C. (1993). The neural basis of drug craving: an incentivesensitization theory of addiction. Brain Research Reviews, 18, 247-291. doi:10.1016/0165-0173(93)90013-P

Rothbart, M. K., Ahadi, S. A., \& Evans, D. E. (2000). Temperament and personality: Origins and outcomes. Journal of Personality and Social Psychology, 78, 122-135. doi: $10.1037 / / 0022-3514.78 .1 .122$

Saunders, J. B., Assland, O. G., Babor, T. F., De la Fuente, J. R., \& Grant, M. (1993).

Development of the alcohol use disorders identification tests (AUDIT): WHO collaborative project on early detection of persons with harmful alcohol consumption-II. Addiction, 88, 791-804. doi:10.1111/j.1360-0443.1993.tb02093.x

Sharma, L., Markon, K. E., Clark, L. A. (2014). Toward a theory of distinct types of “impulsive" behaviors: A meta-analysis of self-report and behavioral measures. Psychological Bulletin, 140(2), 374-408. doi:10.1037/a0034418 
ALCOHOL-RELATED INHIBITORY CONTROL

Sciascia, J. M., Reese, R. M., Janak, P. H., \& Chaudhri, N. (2015). Alcohol-seeking triggered by discrete Pavlovian cues is invigorated by alcohol contexts and mediated by glutamate signaling in the basolateral amygdala. Neuropsychopharmacology, 40, 2801. doi:10.1038/npp.2015.130

Siegel, M. B., Naimi, T. S., Cremeens, J. L., \& Nelson, D. E. (2011). Alcoholic beverage preferences and associated drinking patterns and risk behaviors among high school youth. American Journal of Preventative Medicine, 40, 419-426.

doi:10.1016/j.amepre.2010.12.011

Thrul, J., \& Kuntsche, E. (2015). The impact of friends on young adults' drinking over the course of the evening: An event - level analysis. Addiction, 110, 619-626. doi:10.1111/add.12862

Thrul, J., Labhart, F., \& Kuntsche, E. (2017). Drinking with mixed-gender groups is associated with heavy weekend drinking among young adults. Addiction, 112, 432-439. doi:10.1111/add.13633

Trela, C. J., Hayes, A. W., Bartholow, B. D., Sher, K. J., Heath, A. C., \& Piasecki, T. M. (2018). Moderation of alcohol craving reactivity to drinking-related contexts by individual differences in alcohol sensitivity: An ecological investigation. Experimental and Clinical Psychopharmacology, 26, 354-365. doi:10.1037/pha0000206

Wall, A-M., McKee, S. A., \& Hinson, R. E. (2000). Assessing variation in alcohol outcome expectancies across environmental context: An examination of the situational-specificity hypothesis. Psychology of Addictive Behaviors, 14, 367-375. doi:10.1037/0893164X.14.4.367 
ALCOHOL-RELATED INHIBITORY CONTROL

Weafer, J., \& Fillmore, M. T. (2015). Alcohol-related cues potentiate alcohol impairment of behavioral control in drinkers. Psychology of Addictive Behaviors, 29, 290-299. doi:10.1037/adb0000013

Wiers, R. W., Eberl, C., Rinck, M., Becker, E. S., \& Lindenmeyer, J. (2011). Retraining automatic action tendencies changes alcoholic patients' approach bias for alcohol and improves treatment outcome. Psychological Science, 22, 490-497. doi:10.1177/0956797611400615

Wiers, R. W., Rinck, M., Dictus, M., \& Van den Wildenberg, E. (2009). Relatively strong automatic appetitive action-tendencies in male carriers of the OPRM1 G-allele. Genes, Brain and Behavior, 8, 101-106. doi:10.1111/j.1601-183X.2008.00454.X

Wiers, R. W., Rinck, M., Kordts, R., Houben, K., \& Strack, F. (2010). Retraining automatic action-tendencies to approach alcohol in hazardous drinkers. Addiction, 105, 279-287. doi:10.1111/j.1360-0443.2009.02775.x

Wiers, R. W., Wood, M. D., Darkes, J., Corbin, W. R., Jones, B. T., \& Sher, K. J. (2003). Changing expectancies: Cognitive mechanisms and context effects. Alcohol Clinical \& Experimental Research, 27, 186-197. doi:10.1097/01.ALC.0000051023.28893.8A

Williams, B. J \& Kaufmann, L. M. (2012). Reliability of the Go/No Go Association Task. Journal of Experimental Social Psychology, 48, 879-891. doi:10.1016/j.jesp.2012.03.001

Wright, L., Lipszyc, J., Dupuis, A., Thayapararajah, S. W., \& Schachar, R. (2014). Response inhibition and psychopathology: A meta-analysis of go/no-go task performance. Journal of Abnormal Psychology, 123, 429-439. doi:10.1037/a0036295 